\title{
ON TAILS AND DOMAINS OF ATTRACTION OF STABLE MEASURES IN BANACH SPACES ${ }^{1}$
}

\author{
BY \\ ALOISIO ARAUJO ${ }^{2}$ AND EVARIST GINE ${ }^{3}$
}

\begin{abstract}
The exact tail behavior of stable measures in Banach spaces and measures in their domains of attraction is given. Conditions for a p.m. to be in the domain of attraction of a stable p.m. of order $\alpha$ are derived which are sufficient in type $p$ spaces, $p>\alpha$, and necessary in general. This paper also contains a short proof of the Levy-Khinchin formula in Banach spaces.
\end{abstract}

1. Introduction. Let $B$ be a separable Banach space and $\rho$ a stable law on $(B, \mathscr{B})$, i.e. $\rho$ is such that if $X_{i}$ are i.i.d. with $\mathcal{L}\left(X_{i}\right)=\rho$, then for every $n$ there exist $a_{n}$ and $b_{n}$ such that $\mathcal{L}\left(\sum_{i=1}^{n} X_{i} / a_{n}-b_{n}\right)=\mathfrak{L}(X)$. It is well known that the only possible values for $a_{n}$ are $n^{1 / \alpha}, \alpha \in(0,2]$. We then call $\alpha$ the index or order of $\rho$. A probability measure $\lambda$ belongs to the domain of attraction of $\rho$ if and only if there exist $\left\{a_{n}\right\} \subset \mathbf{R}$ and $\left\{b_{n}\right\} \subset B$ such that whenever $X_{i}$ are i.i.d. with $\mathcal{L}\left(X_{i}\right)=\lambda$, then $\mathcal{L}\left(\sum_{i=1}^{n} X_{i} / a_{n}-b_{n}\right) \rightarrow_{\mathrm{w}} \rho$. It is also well known that only stable measures have nonvoid domains of attraction (Kumar and Mandrekar (1972)). In case the constants $a_{n}$ can be chosen to be $a_{n}=n^{1 / \alpha}, \alpha$ the order of $\rho$, then $\lambda$ is in domain of normal attraction of $\rho$.

The tail behavior of stable measures and measures in their domain of attraction plays an important role in the study of domains of attraction on the line (see e.g. Feller (1970)) and in other spaces as well (Kuelbs and Mandrekar (1974) among others). The tail behavior of a stable p.m. is in turn completely determined by its associated Lévy measure. In this paper we present results on these subjects.

In $\$ 2$ we give a proof of the Lévy-Khinchin representation of infinitely divisible laws in Banach space. Proofs of this theorem have been given independently by Araujo (1975) and Dettweiler (1976). The proof given here

Received by the editors August 23, 1977 and, in revised form October 30, 1977.

AMS (MOS) subject classifications (1970). Primary 60B10; Secondary 60B99.

Key words and phrases. Domains of attraction, stable laws, tails of stable laws, Lévy-Khinchin representation.

'Part of this work was communicated by the authors to the Second Vilnius Conference on Probability and Mathematical Statistics, Vilnius, June 27-July 4, 1977.

${ }^{2}$ This author's research has been partially supported by NSF Grant MP574-18967.

${ }^{3}$ This author's research has been partially supported by CONICIT (Venezuela) Grant 51-26. S1-0893. 
seems to be simpler and does not rely on the finite dimensional result.

In $\$ 3$ we give the exact tail behavior of stable laws and laws in their domains of attraction, thus extending a classical result of P. Lévy (1937) to stable p.m.'s on Banach spaces. This question has been treated in a more general setting by de Acosta (1977); the result in this section refines his in the particular case of a Banach space.

$\$ 4$ contains several results on domains of attraction; we give conditions for a p.m. to be in the domain of attraction of a stable law which are sufficient in type $p$ spaces and necessary in general Banach spaces. Some of these generalize previous work by Kuelbs and Mandrekar (1974).

The methods of this paper are based on the properties of slowly varying functions (as e.g. in Feller (1970) or Ibragimov and Linnik (1971)) and results of Le Cam (1970), and de Acosta, Araujo and Giné (1977) on tightness of row sums in infinitesimal arrays, tightness of generalized Poisson p.m.'s and the relation between tightness of sums and tightness of the accompanying Poisson laws.

In what follows we will only consider separable Banach spaces. The Borel $\sigma$-algebra of a Banach space $B$ will be denoted by $\mathscr{B}$ as usual and the set of Borel p.m.'s on $(B, \mathscr{B})$ by $\mathscr{P}(B)$. All the measures considered here will be Borel measures.

2. The Lévy-Khinchin formula and the representation of stable p.m.'s. We recall that a Lévy measure $\mu$ on $B$ is a positive measure with $\mu\{0\}=0$ such that the function on $B^{\prime}$ to $C$ defined as

$$
\exp \left\{\int\left(e^{i f(x)}-1-i \min (1,\|x\|)\|x\|^{-1} f(x)\right) d \mu(x)\right\}
$$

is the characteristic function of a p.m. on $B$ (Araujo (1975a)). The p.m. with ch.f. given by (2.1) is the centered Poisson p.m. with associated Lévy measure $\mu$, cPois $\mu$. For other equivalent definitions of Lévy measures we refer to de Acosta, Araujo and Giné (1977). In particular $\mu$ is a Lévy measure if and only if $\mu \mid\{\|x\|>\delta\}$ is finite for every $\delta>0$ and $\left\{\operatorname{Pois}\left(\mu \mid\|x\|>\delta_{n}\right)\right\}_{n=1}^{\infty}$ relatively shift compact for some sequence $\delta_{n} \downarrow 0$. (Given a finite positive measure $\tau$, Pois $\tau=e^{-\tau(B)} \sum_{n=0}^{\infty} \tau^{n} / n$ ! where $\tau^{n}=\tau * \cdots * \tau$.)

The main ingredients in our proof are the following two lemmas.

2.1. LemMA. If $\left\{\mu_{\alpha}\right\}_{\alpha \in A}$ is a family of Lévy measures on $B$ such that $\left\{\right.$ cPois $\mu_{\alpha}$ \} is relatively shift compact then

(i) the family of finite measures $\left\{\mu_{\alpha} \mid\|x\|>\delta\right\}_{\alpha \in A}$ is relatively compact for every $\delta>0$.

(ii) $\sup _{\alpha} \int_{\|x\|<1} f^{2}(x) d \mu_{\alpha}(x)<\infty$ for every $f \in B^{\prime}$.

For a proof we refer to Parthasarathy (1967), IV. 4.3, or to de Acosta, Araujo, Giné (loc. cit.), Theorem 1.4. 
A family of random variables $\left\{X_{n j}: j=1, \ldots, k_{n}, n=1, \ldots\right\}$ is an infinitesimal array if for each $n$ the variables $X_{n 1}, \ldots, X_{n k_{n}}$ are independent and for every $\delta>0, \lim _{n \rightarrow \infty} \max P\left\{\left\|X_{n j}\right\|>\varepsilon\right\}=0$. The following lemma is due to Le Cam (1970). For a proof see de Acosta, Araujo and Giné (loc. cit).

2.2. LemMA. Let $\left\{X_{n j}\right\}$ be an infinitesimal system of $B$ valued rv's and $S_{n}=\sum_{j=1}^{k_{n}} X_{n j}$. Then if $\left\{\mathcal{L}\left(S_{n}\right)\right\}$ is relatively shift compact, there exists for every $\delta>0$ a compact convex symmetric set $K_{\varepsilon}$ of diameter at most $\varepsilon$ such that the family of measures $\left\{\sum_{j=1}^{k_{n}} \mathcal{L}\left(X_{n j}\right) \mid K_{\varepsilon}^{c}\right\}_{n=1}^{\infty}$ is relatively compact and

$$
\lim _{n \rightarrow \infty} \max _{j} P\left\{X_{n j} \in K_{\varepsilon}^{c}\right\}=0 .
$$

We recall that a p.m. $\rho$ is infinitely divisible if for every natural $n$ there exists a p.m. $\rho_{n}$ such that $\rho=\rho_{n} * \cdots * \rho_{n}$ ( $n$ times). We call $\rho_{n}$ the $n$th root of $\rho$. The following lemma is also needed:

2.3. LEMMA. If $\rho$ is infinitely divisible then

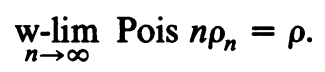

For the proof see de Acosta and Samur (1977).

Now we can prove the theorem.

2.4. THEOREM. $\rho \in \mathscr{P}(B)$ is infinitely divisible if and only if there exist $x_{0} \in B, a$ centered Gaussian p.m. $\gamma$ and a Lévy measure $\mu$ with $\mu\{0\}=0$ such that

$$
\rho=\delta_{x_{0}} * \gamma * \text { cPois } \mu .
$$

$x_{0}, \gamma$ and $\mu$ are unique, and the characteristic function of $\rho$ is

$$
\begin{aligned}
\hat{\rho}(f)=\exp \{ & i f\left(x_{0}\right)-\frac{1}{2} \Phi_{\gamma}(f, f) \\
& \left.+\int\left(e^{i f(x)}-1-i \min (1,\|x\|)(f(x) /\|x\|) d \mu(x)\right)\right\} .
\end{aligned}
$$

Proof. By Lemma 2.3 Pois $n \rho_{n} \rightarrow_{\mathrm{w}} \rho$. By Lemma 2.1(i) it is possible to construct, using a diagonalisation argument, a $\sigma$-finite measure $\mu$ with $\mu\{0\}=$ 0 such that for some subsequence $\left\{n^{\prime}\right\}$ and every $\varepsilon>0$ satisfying $\mu\{\|x\|=\varepsilon\}$ $=0$,

$$
n^{\prime} \rho_{n^{\prime}}|\{\|x\|>\varepsilon\} \underset{\mathbf{w}}{\rightarrow} \mu|\{\|x\|>\varepsilon\} .
$$

For every such $\varepsilon>0$ there exists then $n_{\varepsilon}^{\prime}$ such that

$$
d_{p}\left[\operatorname{Pois}\left(n_{\varepsilon}^{\prime} \rho_{n_{\varepsilon}^{\prime}} \mid\|x\|>\varepsilon\right), \operatorname{Pois}(\mu \mid\|x\|>\varepsilon)\right]<\varepsilon,
$$

where $d_{p}$ is Prokhorov's distance. Obviously we may assume $n_{\varepsilon} \uparrow \infty$ as $\varepsilon \downarrow 0$. Since $\left\{\operatorname{Pois}\left(n_{\varepsilon}^{\prime} \rho_{n_{\varepsilon}^{\prime}} \mid\|x\|>\varepsilon\right)\right\}$ is relatively shift compact (as it is a set of factors of the tight family $\left\{\right.$ Pois $\left.\left.n \rho_{n}\right\}\right)$, so is $\{\operatorname{Pois}(\mu \mid\|x\|>\varepsilon)\}$ and therefore, $\mu$ is a 
Lévy measure. Also, if $\varepsilon_{r} \downarrow 0$ with $\mu\left\{\mid\|x\|=\varepsilon_{r}\right\}=0$, there exist $a_{r} \in B$ such that $\delta_{a_{r}} * \operatorname{Pois}\left(n_{r} \rho_{n_{r}} \mid\|x\|>\varepsilon_{r}\right) \rightarrow_{\mathrm{w}}$ cPois $\mu$ where for ease of notation we write $n_{r}$ for $n_{\varepsilon_{r}}^{\prime}$. This, together with the convergence of $\left\{\right.$ Pois $\left.n \rho_{n}\right\}$, gives the tightness of $\left\{\delta_{-a_{r}} *\right.$ Pois $\left.n_{r} \rho_{n_{r}} \mid\|x\| \leqslant \varepsilon_{r}\right\}$. By taking a subsequence if necessary, we may assume that $\delta_{-a_{r}} * \operatorname{Pois}\left(n_{r} \rho_{n_{r}} \mid\|x\| \leqslant \varepsilon_{r}\right) \rightarrow_{\mathrm{w}} \tau$. Next we prove that $\tau$ is Gaussian.

The logarithms of the ch.f.'s of these p.m.'s are

$$
n_{r} \int_{\|x\|<\varepsilon_{r}}(\cos f(x)-1) d \rho_{n_{r}}(x)+i n_{r} \int_{\|x\|<\varepsilon_{r}}\left(f\left(-a_{r}\right)+\sin f(x)\right) d \rho_{n_{r}}(x) .
$$

By Lemma 2.1(ii)

$$
\sup _{r} n_{r} \int_{\|x\|<\varepsilon_{r}} f^{2}(x) d \rho_{n_{r}}(x)<\infty
$$

and therefore, since $\cos f(x)-1+f^{2}(x) / 2 \approx c f^{4}(x)$ and $|\sin f(x)-f(x)| \approx$ $c^{\prime}|f(x)|^{3}$ as $f(x) \rightarrow 0$, the sequence above, which converges (Chung (1974), Theorem 7.6.3) has the same limit as the sequence $\left\{-2^{-1} n_{r} \int_{\|x\|<r_{r}} f^{2}(x) d \rho_{n_{r}}(x)\right.$ $\left.+i n_{r} \int f\left(x-a_{r}\right) d \rho_{n_{r}}(x)\right\}$.

Hence for each $f \in B^{\prime}, \hat{\tau}(f)=e^{-\Phi(f f)+i a_{1}}$, i.e. $\tau \circ f^{-1}$ is Gaussian (not necessarily centered). Then, by symmetrisation one sees that $e^{-\Phi(f, f)}$ is the ch.f. of a centered Gaussian p.m. $\gamma$. Therefore the linear functional $a_{f}$ is weak-star sequentially continuous (as $\hat{\tau}$ and $\hat{\gamma}$ are) thus defining a point $a$ in $B$ by the equation $f(a)=a_{f}$ (Schaefer (1973), Corollary IV.6.2.3).

Hence

$$
\rho=\delta_{a} * \gamma * \text { cPois } \mu \text {. }
$$

The unicity of the decomposition follows from the proof of Theorem IV.8.1 in Parthasarathy (1967).

As a corollary one obtains the following representation for stable p.m.'s.

2.5. Corollary. Let $\rho$ be a stable p.m. on B. If $\rho$ is of order $\alpha<2$ then

$$
\rho=\delta_{x_{0}} * \text { cPois } \mu
$$

where $\mu$ has the following form: there exists a finite measure $\sigma$ on the set $S=\{x:\|x\|=1\}$ such that if $r(x)=\|x\|$ and $s(x)=x /\|x\|$, then

$$
d \mu(r, s)=r^{-1-\alpha} d r d \sigma(s) .
$$

If $\alpha=2$ then $\rho$ is a Gaussian p.m.

Proof. Exactly as in Kuelbs (1973), p. 261.

3. Tails of measures in the domain of attraction of stable laws. Let $\rho$ be a nondegenerate stable measure on $B, \mu$ its associated Lévy measure and let $X$ be a random variable in the domain of attraction of $\rho$ with associated sequences $\left\{a_{n}, b_{n}\right\}$, i.e. such that if $\left\{X_{i}\right\}_{i=1}^{\infty}$ are i.i.d. with $\mathcal{L}\left(X_{i}\right)=\mathcal{L}(X)$, then 
$\mathcal{L}\left(\sum_{i=1}^{n} X_{i} / a_{n}-b_{n}\right) \rightarrow_{\mathrm{w}} \rho$. Then,

3.1.THEOREM. For every $\delta>0$,

$$
n \mathcal{L}\left(X / a_{n}\right)|\{\|x\|>\delta\} \underset{\mathrm{w}}{\rightarrow} \mu|\{\|x\|>\delta\} .
$$

For the proof we need a simple measure theoretic lemma.

3.2. LemMA. Let $B$ be a separable Banach space, $\mathscr{B}^{\prime}$ the $\sigma$-ring of Borel subsets of $B$ not containing zero and $\mathcal{U}^{\prime}$ the $\sigma$-ring generated by the ring of cylindrical sets at a positive distance from zero. Then $\mathscr{B}^{\prime}=\mathcal{U}^{\prime}$.

PRoof. It is well known that $\{\|x\|>r\}=\cup_{i=1}^{\infty}\left\{\left|f_{i}(x)\right|>r\right\}$ for some countable family $\left\{f_{i}\right\} \subset B^{\prime}$. So, $\{\|x\|>r\} \in \mathcal{U}^{\prime}$. If $\pi$ is a continuous finite dimensional projection then

$$
\pi^{-1}\{0\} \backslash\{0\}=\bigcap_{r} \bigcup_{n}\left[\pi^{-1}\left\{\|\pi(x)\| \leqslant r^{-1}\right\} \cap\left\{\|x\|>n^{-1}\right\}\right]
$$

so that $\pi^{-1}\{0\} \backslash\{0\} \in \mathcal{U}^{\prime}$. Hence, if $A$ is a Borel set in $\pi(B), \pi^{-1}(A) \backslash\{0\}$ $=\left[\cup_{n} \pi^{-1}\left(A \cap\left\{\|y\|>n^{-1}\right\}\right)\right] \cup\left[\pi^{-1}(A \cap\{0\}) \backslash 0\right] \in \mathcal{U}^{\prime}$. So $\mathcal{U}^{\prime}$ contains all the cylinder sets deprived of $\{0\}$, and therefore the $\sigma$-algebra $\left\{B: B \in \mathcal{U}^{\prime}\right.$ or $B=C \cup\{0\}$ with $\left.C \in \mathscr{Q}^{\prime}\right\}$ contains the cylinder sets i.e. is the Borel $\sigma$-algebra. This proves that $\mathfrak{B}^{\prime}=\mathcal{U}^{\prime}$.

Proof of THEOREM 3.1. It is easy to prove that if $X$ is nondegenerate then $a_{n} \rightarrow \infty$ and therefore the system $\left\{X_{n j}=X_{j} / a_{n}: j=1, \ldots, n=1, \ldots\right\}$ is infinitesimal. Since the sums are shift tight, Lemma 2.2 implies that the sequence $\left\{n \mathcal{L}\left(X / a_{n}\right) \mid\|x\|>\delta\right\}$ is relatively compact for every $\delta>0$. Let $\nu^{1}$ be a limit of this sequence for $\delta=1$. Then, by a simple diagonal argument we can construct a $\sigma$-finite measure $\nu$ on $B$, with $\nu\{0\}=0$ and $\nu \mid\{\|x\|>1\}=$ $\nu^{1}$ such that for some sequence $\left\{n^{\prime}\right\} \subset\{n\}, n^{\prime} \rightarrow \infty$, and $\delta_{k} \downarrow 0$, $n^{\prime} \mathcal{L}\left(X / a_{n^{\prime}}\right)\left|\left\{\|x\|>\delta_{k}\right\} \rightarrow_{\mathrm{w}} \nu\right|\left\{\|x\|>\delta_{k}\right\}$. If $\nu\left|\left\{\|x\|>\delta_{k}\right\} \neq \mu\right|\left\{\|x\|>\delta_{k}\right\}$ for some $k$ then by the previous lemma, there exists a cylinder set $A$ at a positive distance from zero with $\nu(A) \neq \mu(A)$. Moreover, $A$ may be assumed to be a continuity set for both $\mu$ and $\nu$. Let $\pi$ be a finite dimensional projection such that $A=\pi^{-1}(C)$ with $C$ at a positive distance from zero in the Euclidean distance on the range of $\pi$. Since the system $\left\{\pi\left(X_{n j}\right)\right\}$ is infinitesimal and $\left\{\mathcal{L}\left(\sum_{j=1}^{k_{n}} \pi\left(X_{n j}\right)\right)\right\}$ is shift convergent, the finite dimensional central limit theorem implies $\sum_{j=1}^{n} P\left\{\pi\left(X_{n j}\right) \in C\right\} \rightarrow \mu(A)$; but if $A \cap\{\|x\|$ $\left.\leqslant \delta_{k}\right\}=\varnothing$ for some $k$, then

$$
\sum_{j=1}^{n} P\left\{\pi\left(X_{n j}\right) \in C\right\}=n\left[\mathcal{L}\left(X / n^{1 / \alpha}\right) \mid\left\{\|x\|>\delta_{k}\right\}\right](A) \rightarrow \nu(A) ;
$$

therefore $\mu(A)=\nu(A)$, contradiction. Hence (3.1) is proved for every $\delta>0$ such that $\mu\{\|x\|=\delta\}=0$, but by the particular form of $\mu$ this is true for all $\delta>0$. 
3.2 COROLLARY. If $X$ is a stable $B$-valued $r v$ of index $\alpha \in(0,2)$ or it is in the normal domain of attraction of a stable law of index $\alpha$ having Lévy measure $\mu$ then for every $\delta>0$

$$
n \mathcal{L}\left(X / n^{1 / \alpha}\right)|\{\|x\|>\delta\} \underset{\mathrm{w}}{\rightarrow} \mu|\{\|x\|>\delta\} .
$$

In particular, $\lim _{n \rightarrow \infty} n P\left\{X /\|X\| \in A,\|X\|>\delta n^{1 / \alpha}\right\}=\sigma(A) / \alpha \delta^{\alpha}$ and $E\|X\|^{p}<\infty$ for every $p<\alpha$.

This corollary is a complete generalization of Lévy's result on tails of stable p.m.'s on the line. It was obtained by Kuelbs and Mandrekar (1974) for Hilbert spaces. De Acosta (1977) has weaker statements in a more general setting.

3.3. Remark. Corollary 3.2 extends to the case $\alpha=2$ : if $X$ is in the normal domain of attraction of a Gaussian law, we have

$$
n \mathcal{L}\left(X / n^{1 / 2}\right) \mid\{\|x\|>\delta\} \underset{\mathrm{w}}{\rightarrow} 0
$$

for every $\delta>0$ (de Acosta, Araujo, Giné (loc. cit.)).

If $X$ is a real variable in the domain of attraction of a stable law of order $\alpha \in(0,2)$ then the function $t^{\alpha} P\{|X|>t\}$ is slowly varying. This result has been extended by Kuelbs and Mandrekar (1974) to Hilbert space valued rv's. As another corollary to Theorem 3.1 we show that it is true in separable Banach spaces as well.

3.4. Corollary. If $X$ is a B-valued $r v$ in the domain of attraction of a nondegenerate stable law of order $\alpha \in(0,2)$, then the function $t^{\alpha} P\{\|X\|>t\}$ is slowly varying.

Proof. Let $X$ belong to the domain of attraction of a nondegenerate stable p.m. $\rho$ of order $\alpha$ with Lévy measure $\mu$. Then for every $t, u>0$,

$$
\mu\{\|x\|>t\} / \mu\{\|x\|>t u\}=\int_{t}^{\infty} r^{-\alpha-1} d r / \int_{t u}^{\infty} r^{-\alpha-1} d r=u^{\alpha} .
$$

If $a_{n} \rightarrow \infty$ is a set of constants such that $\left\{\mathcal{L}\left(X_{1}+\cdots+X_{n}\right) / a_{n}\right\}$ is weakly shift convergent, define $n_{t}$ as the largest $n$ such that $a_{n} \leqslant t$. Note that if $t \rightarrow \infty$ so does $\left\{n_{t}\right\}$. Then,

$$
\begin{aligned}
{\left[n_{t} /\left(n_{t}+1\right)\right] } & \left(n_{t}+1\right) P\left\{\|X\|>a_{n_{t}+1}\right\} / n_{t} P\left\{\|X\|>a_{n_{t}} u\right\} \\
& <P\{\|X\|>t\} / P\{\|X\|>t u\} \\
& \leqslant\left[\left(n_{t}+1\right) / n_{t}\right] n_{t} P\left\{\|X\|>a_{n_{t}}\right\} /\left(n_{t}+1\right) P\left\{\|X\|>a_{n_{t}+1} u\right\} .
\end{aligned}
$$

Letting $t \rightarrow \infty$, Theorem 3.1 and the previous computation give that for every $u>0$,

$$
\lim _{t \rightarrow \infty} P\{\|X\|>t\} / P\{\|X\|>t u\}=u^{\alpha},
$$

i.e. that the function $P\{\|X\|>t\}$ is regularly varying of index $-\alpha$. Hence, 
the function $t^{\alpha} P\{\|X\|>t\}$ is slowly varying.

4. Domains of attraction. The proofs of the results we present in this section can be done directly. However we will base them on the following two theorems.

4.1. Lemma. Let $\left\{X_{n j}: j=1, \ldots, k_{n}, n=1, \ldots\right\}$ be a family of row-wise independent $B$-random variables, $S_{n}=\sum_{j=1}^{k_{n}} X_{n j}$ and

$$
b_{n}=\sum_{j=1}^{k_{n}} \int \min (1,\|x\|)\|x\|^{-1} x d \mathfrak{L}\left(X_{n j}\right)(x) .
$$

Then if $\left\{\text { Pois } \sum_{j=1}^{k_{n}} \mathcal{L}\left(X_{n j}\right)\right\}_{n=1}^{\infty}$ is relatively shift compact, the sequence $\left\{\mathscr{L}\left(S_{n}-\right.\right.$ $\left.\left.b_{n}\right)\right\}$ is relatively compact.

For a proof of this lemma we refer to de Acosta, Araujo and Giné (loc.cit).

4.2. LemMA. Let $\left\{\mu_{\alpha}\right\}_{\alpha \in A}$ be a set of positive Borel measures on a separable Banach space $B$ of Rademacher type $p \in[1,2]$ such that

(i) $\left\{\mu_{\alpha} \mid\|x\|>1\right\}_{\alpha \in A}$ is relatively compact,

(ii) for every $f \in B^{\prime}, \sup _{\alpha \in A} \int_{\|x\|<1} f^{2}(x) d \mu_{\alpha}(x)<\infty$,

(iii) there exist an increasing sequence of finite dimensional subspaces $F_{n}$ such that

$$
\lim _{n \rightarrow \infty} \sup _{\alpha \in A} \int_{\|x\|<1} d^{p}\left(x, F_{n}\right) d \mu_{\alpha}(x)=0 .
$$

Then every $\mu_{\alpha}$ is a Lévy measure on $B$ and $\left\{\mathrm{cPois} \mu_{\alpha}\right\}_{\alpha \in A}$ is relatively compact.

For the definition of a type $p$ Rademacher space see e.g. Hoffman-Jørgensen and Pisier (1976) or references there. The proof of the previous lemma is contained in de Acosta, Araujo and Giné (loc. cit.).

Obviously, combinations of 4.1 and 4.2 may give tightness results for row sums of triangular arrays. This is what we do next.

The following theorem characterizes normal domains of attraction.

4.3. TheOREM. (i) If $B$ is of type $p \in[1,2]$ and $\rho$ is a stable law of index $\alpha<p$ then a sufficient condition for a B-valued ro $X$ to belong to the normal domain of attraction of $\rho$ is that:

(a) $\pi(X)$ belong to the domain of normal attraction of $\rho \circ \pi^{-1}$ for every linear continuous map $\pi$ on $B$ with finite dimensional range,

(b) for every $\delta>0, \sup _{n} n P\left\{\|X\|>\delta n^{1 / \alpha}\right\}<\infty$,

(c) there exist an increasing sequence of finite dimensional subspaces $F_{m} \subset B$ such that

$$
\lim _{m \rightarrow \infty} \limsup _{n \rightarrow \infty} n P\left\{d\left(X, F_{m}\right)>t n^{1 / \alpha}\right\}=0 \text { for some } t>0 .
$$

(ii) The converse is true in any (separable) Banach space. 
Proof. Direct part. We prove first shift tightness of $S_{n} / n^{1 / \alpha}=$ $\sum_{i=1}^{n} X_{i} / n^{1 / \alpha}, X_{i}$ i.i.d. with $\mathcal{L}\left(X_{i}\right)=\mathfrak{L}(X)$. There is no loss of generality in assuming $t=1$ and changing lim $\sup _{n}$ by $\sup _{t \in \mathbf{R}_{+}}$in (4.1), i.e.

$$
\lim _{m \rightarrow \infty} \sup _{t>0} t P\left\{d\left(X, F_{m}\right)>t^{1 / \alpha}\right\}=0 .
$$

Let us set $C_{m}=\sup _{t>0} t P\left\{d\left(X, F_{m}\right)>t^{1 / \alpha}\right\}$.

First we note that the family of finite measures $\left\{n \mathcal{L}\left(X / n^{1 / \alpha}\right) \mid\|x\|>\delta\right\}$ is relatively compact for every $\delta>0$ : by $(4.1)^{\prime}$ these measures, which are uniformly bounded (hypothesis (b)), are flatly concentrated, and by (a) and the converse central limit theorem in the line,

$$
\lim _{m \rightarrow \infty} \limsup _{n \rightarrow \infty} n P\left\{\left|f(X) / n^{1 / \alpha}\right|>m\right\}=0
$$

for every $f \in B^{\prime}$, i.e. all their one dimensional marginals are tight. So, the tightness of $\left\{n \mathcal{L}\left(X / n^{1 / \alpha}\right) \mid\|x\|>\delta\right\}$ follows from a result of de Acosta (1970).

Also,

$$
\lim _{m \rightarrow \infty} \sup _{n} n^{1-p / \alpha} \int_{\|X\|<n^{1 / \alpha}} d^{p}\left(X, F_{m}\right) d P=0 .
$$

In fact, if $\tau_{m}=\mathcal{L}\left(d\left(X, F_{m}\right)\right)$,

$$
\begin{aligned}
& n^{1-p / \alpha} \int_{\|X\|<n^{1 / \alpha}} d^{p}\left(X, F_{m}\right) d P \leqslant n^{1-p / \alpha} p \int_{0}^{n^{1 / \alpha}} \int_{0}^{x} y^{p-1} d y d \tau_{m}(x) \\
& \leqslant n^{1-p / \alpha} p \int_{0}^{n^{1 / \alpha}} C_{m} y^{p-1-\alpha} d y=C_{m} p /(p-\alpha) \rightarrow 0
\end{aligned}
$$

uniformly in $n$.

The same type of computation shows that by (a), for every $f \in B^{\prime}$,

$$
\sup _{n} n^{1-2 / \alpha} \int_{\|X\|<n^{1 / \alpha}} f^{2}(X) d P<\infty .
$$

Now, the tightness of $\left\{n \mathcal{L}\left(X / n^{1 / \alpha}\right) \mid\|x\|>\delta\right\}$, (4.2) and (4.3) imply by Lemma 4.2 that $\left\{\right.$ Pois $\left.n \mathcal{L}\left(X / n^{1 / \alpha}\right)\right\}$ is relatively shift compact and therefore that $\left\{\mathcal{L}\left(S_{n} / n^{1 / \alpha}-b_{n}\right)\right\}$, with $b_{n}=n \int \min (1,\|x\|)\|x\|^{-1} x d \mathcal{L}\left(X / n^{1 / \alpha}\right)(x)$, is relatively compact by Lemma 4.1.

Suppose now that $\left\{n \mathcal{L}\left(X / n^{1 / \alpha}\right) \mid\|x\|>\delta\right\}$ has two limits $\mu^{\delta}$ and $\nu^{\delta}$. Then by a diagonal argument we can find $\mu, \nu \sigma$-finite, such that $\mu\{0\}=\nu\{0\}=0$, $\mu^{\delta}=\mu\left|\{\|x\|>\delta\}, \nu^{\delta}=\nu\right|\{\|x\|>\delta\}$, subsequences $\left\{n^{\prime}\right\},\left\{n^{\prime \prime}\right\}$ and $\delta_{k} \downarrow 0$ such that $n^{\prime} \mathcal{L}\left(X / n^{\prime 1 / \alpha}\right)\left|\left\{\|x\|>\delta_{k}\right\} \rightarrow_{w} \mu\right|\left\{\|x\|>\delta_{k}\right\}$ and $n^{\prime \prime} \mathcal{L}\left(X / n^{\prime \prime 1 / \alpha}\right)\left|\left\{\|x\|>\delta_{k}\right\} \rightarrow_{\mathrm{w}} \nu\right|\left\{\|x\|>\delta_{k}\right\}$. Now, if $\mu \neq \nu$ this implies by 3.1 that $\mu(A) \neq \nu(A)$ for some cylinder set $A=\pi^{-1}(C)$ at a positive distance from 0 such that $\mu(\partial A)=\nu(\partial A)=0$; but by (a) and the finite dimensional 
central limit theorem,

$$
\begin{aligned}
\mu(A) & =\lim _{n^{\prime} \rightarrow \infty} n^{\prime} P\left\{X / n^{\prime 1 / \alpha} \in A\right\} \\
& =\lim _{n^{\prime \prime} \rightarrow \infty} n^{\prime \prime} P\left\{X / n^{\prime \prime 1 / \alpha} \in A\right\}=\nu(A),
\end{aligned}
$$

contradiction. So, $\mu=\nu$ and $\left\{n \mathcal{L}\left(X / n^{1 / \alpha}\right) \mid\|x\|>\delta\right\}$ converges. Hence, if for any $\left(f_{1}, \ldots, f_{n}\right) \in B^{\prime}$ we let

$$
\begin{aligned}
b_{m}\left(f_{1}, \ldots, f_{n}\right)=m \int \min \left(1,\left|\left(f_{1}, \ldots, f_{n}\right)(x)\right|\right)\left|\left(f_{1}, \ldots, f_{n}\right)(x)\right|^{-1} \\
\cdot\left(f_{1}, \ldots, f_{n}\right)(x) d \mathcal{L}\left(X / m^{1 / \alpha}\right)(x)
\end{aligned}
$$

where $\left|\left(f_{1}, \ldots, f_{n}\right)(x)\right|=\left(\sum_{i=1}^{n} f_{i}^{2}(x)\right)^{1 / 2}$, we obtain that $\left\{\left(f_{1}\left(b_{m}\right), \ldots, f_{n}\left(b_{m}\right)\right)\right.$ $\left.-b_{m}\left(f_{1}, \ldots, f_{n}\right)\right\}_{m=1}^{\infty}$ is a convergent sequence: it is the integral with respect to $m \mathcal{L}\left(X / \mathrm{m}^{1 / \alpha}\right)$ of a bounded continuous function vanishing inside a neighborhood of zero. Hence, since $b_{m}\left(f_{1}, \ldots, f_{n}\right)$ is the natural centering of $\left(f_{1}, \ldots, f_{n}\right)\left(S_{m} / m^{1 / \alpha}\right)$, we conclude that $\left\{\mathcal{L}\left(S_{n} / n^{1 / \alpha}-b_{n}\right)\right\}$ is not only tight, but all its finite dimensional distributions converge. The finite dimensional limits are shifts of projections of $\rho$ and therefore $\left\{\mathcal{L}\left(S_{n} / n^{1 / \alpha}-b_{n}\right)\right\}$ converges to a shift of $\rho$.

(ii) If $X$ is in the domain of normal attraction of $\rho$, (a) follows trivially and (b) is part of Theorem 3.1. Let us prove (c). Let $\mu$ be the Lévy measure associated to $\rho$. Its particular form (Corollary 2.5) implies that

(1) for every Borel set $B$ at a positive distance from zero and $u>0$,

$$
\mu(u B)=u^{-\alpha} \mu(B)
$$

(as a simple change of variables shows),

(2) by Fubini's theorem, $\mu\{d(x, F)=a\}=0$ for every $a>0$ and measurable vector space $F \subset B$.

Then, Theorem 3.1 gives

$$
\lim _{n \rightarrow \infty} n P\left\{d(X, F)>n^{1 / \alpha} t\right\}=c t^{-\alpha}
$$

where $c=\mu\{d(x, F)>1\}$. Since $\mu$ is finite outside the origin, whenever $F_{m} \uparrow$, $\overline{\cup F_{m}}=B$, we get $c_{m}=\mu\left\{d\left(x, F_{m}\right)>1\right\} \rightarrow 0$ and (c) is a consequence of the previous limit.

4.4. REMARK. If $X$ and $\rho$ are symmetric in Theorem 4.3, then condition (a) can be simplified: it is enough to assume instead $\left(a^{\prime}\right)$ the random variables $f(X), f \in B^{\prime}$, belong to the normal domain of attraction of $\rho \circ f^{-1}$. Proof: one obtains that $\left\{\mathcal{L}\left(S_{n} / n^{1 / \alpha}\right)\right\}$ is shift tight as in the above proof, but by symmetry this sequence is tight; by $\left(\mathrm{a}^{\prime}\right) \mathcal{L}\left(f\left(S_{n}\right) / n^{1 / \alpha}\right) \rightarrow_{\mathrm{w}} \rho \circ f^{-1}$, hence $\mathcal{L}\left(S_{n} / n^{1 / \alpha}\right) \rightarrow_{\mathrm{w}} \rho$ (note that by the symmetry of $X$ and $\rho$ the shift of $f\left(S_{n}\right) / n^{1 / \alpha}$ is zero).

4.5. ReMARK. In case the space $B$ has an increasing sequence $\left\{\pi_{m}\right\}$ of projections with finite dimensional range converging pointwise to the identity, 
condition (c) can be replaced by

$$
\lim _{m \rightarrow \infty} \lim _{n} \sup n P\left\{\left\|\left(I-\pi_{m}\right) X\right\|>t n^{1 / \alpha}\right\}=0 .
$$

4.6. ExAmple. $L_{p}, 2 \leqslant p<\infty$, is of type 2 and therefore the last theorem applies for $B=L_{p}$.

4.7. EXAMPLE. Since every Banach space is of type 1 , the conditions in Theorem 4.3 are necessary and sufficient in any Banach space if $\alpha<1$.

4.8. ExAMPLE. Let $\left\{e_{i}\right\} \subset B$ of type $p$ and $\left\{\varphi_{i}\right\}$ a sequence of independent real symmetric rv's in the domain of normal attraction of a symmetric stable law of index $\alpha<p$. Assume that $X=\sum_{i=1}^{\infty} \varphi_{i} e_{i}$ is well defined and in $\mathscr{B}$ and that

(i) $\lim \sup _{t \rightarrow \infty} t^{\alpha} P\left\{\left\|\sum_{i=m}^{\infty} \varphi_{i} e_{i}\right\|>t\right\}=C_{m}<\infty, m=1, \ldots$, and $C_{m} \rightarrow 0$ as $m \rightarrow \infty$,

(ii) if we let $m_{i}(f)=\lim _{t \rightarrow \infty} t^{\alpha} P\left\{\varphi_{i} f_{i}\left(e_{i}\right)>t\right\}$, then $\sum_{i=1}^{\infty} m_{i}(f)<\infty$ for every $f \in B^{\prime}$.

Then $X$ is in the domain of normal attraction of a symmetric stable law.

In fact, conditions (i) and (ii) allow for the application of the proposition in Feller (1970), p. 278, and to obtain that for every $f \in B^{\prime}$, $\lim _{t \rightarrow \infty} t^{\alpha} P\left\{\sum_{i=1}^{\infty} \varphi_{i} f\left(e_{i}\right)>t\right\}=\sum_{i=1}^{\infty} m_{i}(f)<\infty$. Hence $\sum \varphi_{i} f\left(e_{i}\right)$ is in the domain of normal attraction of a symmetric stable law of order $\alpha$. This together with (ii) gives the tightness of $\left\{\varrho\left(S_{n} / n^{1 / \alpha}\right)\right\}$ as in the proof of Theorem 4.3 (where $S_{n}$ is the sum of $n$ i.i.d. rv's with the law of $\Sigma \varphi_{i} e_{i}$ ); since the one dimensional marginals converge (note that by symmetry the shifts are zero), $\left\{\mathfrak{L}\left(S_{n} / n^{1 / \alpha}\right)\right\}$ is also weakly convergent.

4.9. ReMARK. The case $\alpha=p=2$ is solved in Hoffman-Jørgensen and Pisier (1976): if $E\|X\|^{2}<\infty, E X=0$ and $B$ is of type 2 then $X$ is in the normal domain of attraction of a Gaussian law; conversely if $E\|X\|^{2}<\infty$, $E X=0$ imply that $X$ is in the normal domain of attraction of a Gaussian law, then $B$ is of type 2 . We could ask if the previous theorem admits the same type of converse too.

In the next two theorems we just put together results on tightness of exponentials with lemmas of Kuelbs and Mandrekar (1974) to obtain results on general domains of attraction in certain Banach spaces. Kuelbs and Mandrekar (1974) have interesting necessary and sufficient conditions for $X$ to be in the domain of attraction of a stable measure in Hilbert space although they seem harder to verify than the conditions in Theorem 4.3. Next we state two theorems which are direct generalizations of their results. The proofs follow easily from techniques in their paper and those used so far in this section. Following Kuelbs and Mandrekar (loc. cit.), we will say in what follows that a measure on $B$ is nondegenerate if the closure of the linear span of its topological support coincides with $B$. (Note that in Corollary 3.4 we use 
the term with its usual meaning, namely that the measure is not concentrated on a single point.)

4.10. Theorem. (i) Let $B$ be of type $p \in[1,2], \alpha<p$, and $\rho=\delta_{x} * \operatorname{cPois} \mu$ with $X \in B, d \mu(r, s)=r^{-1-\alpha} d r d \sigma(s), \sigma$ a finite positive nondegenerate measure on $B$ with support contained in $S=\{x:\|x\|=1\}$. Then, for a random variable $X$ to be in the domain of attraction of $\rho$ it is sufficient that:

(a) there exist an increasing sequence of finite dimensional subspaces $F_{m} \subset B$ such that

$$
\lim _{t \rightarrow \infty} P\{\|X\|>t\} / P\left\{d\left(X, F_{m}\right)>t u\right\}=c_{1} u^{\alpha} / C_{m}
$$

for every $u>0$, with $C_{m}>0, C_{m} \rightarrow 0$,

(b) for every $A, D \in \mathscr{B}(S)$, with $\sigma(\partial A)=\sigma(\partial D)=0$ and $\sigma(D) \neq 0$,

$\lim _{t \rightarrow \infty} P\{\|X\|>t, X /\|X\| \in A\} / P\{\|X\|>t, X /\|X\| \in D\}=\sigma(A) / \sigma(D)$.

(ii) In any separable Banach space, conditions (a) and (b) are necessary for $X$ to belong to the domain of normal attraction of $\rho$.

Proof. (i) As in the proof of Lemma 4.1, Kuelbs and Mandrekar (loc. cit.), the conditions (a) and (b) imply the existence of a sequence $\left\{a_{n}\right\}, a_{n}>0$, $a_{n} \uparrow \infty, a_{n} / a_{n+1} \rightarrow 1$, such that:

$$
\lim _{n \rightarrow \infty} n P\left\{\|X\|>t a_{n}, X /\|X\| \in A\right\}=\sigma(A) / \alpha t^{\alpha}
$$

for all continuity sets $A \subset S$ of $\sigma$ and $t>0$,

$$
\begin{array}{ll}
\lim _{m \rightarrow \infty} \sup _{n} n a_{n}^{-p} \int_{\|X\|<\varepsilon a_{n}} d^{p}\left(X, F_{m}\right) d P=0 & \text { for some } \varepsilon>0, \\
\lim _{\varepsilon \downarrow 0} \limsup _{n \rightarrow \infty} n a_{n}^{-2} \int_{|f(X)|<\varepsilon a_{n}} f^{2}(X) d P=0 & \text { for every } f \in B^{\prime} .
\end{array}
$$

(To see this, proceed as in their proof but replace the function $U$ there by $U(t)=\int_{\|X\|<t}\|X\|^{p} d P$.) Since (1) implies that $\left\{n \mathcal{L}\left(X / a_{n}\right) \mid\|x\|>\delta\right\}$ converges weakly to $\mu \mid\{\|x\|>\delta\}$ for every $\delta>0$, Lemma 4.2 gives the tightness of $\left\{\right.$ cPois $\left.\mu_{n}\right\}, \mu_{n}=n \mathcal{L}\left(X / a_{n}\right)$. So, if $\left\{X_{i}\right\}$ are i.i.d. with $\mathcal{L}\left(X_{i}\right)=\mathcal{L}(X)$, then by Lemma 4.1 the sequence $\left\{\mathcal{L}\left(\sum_{i=1}^{n} X_{i} / a_{n}-b_{n}\right)\right\}$ is relatively compact $\left(b_{n}\right.$ as given by Lemma 4.1). On the other hand, (1) and (3) and the finite dimensional central limit theorem imply that for all $\left\{f_{i}\right\}_{i=1}^{m} \subset B^{\prime}$, $\left\{\mathcal{L}\left(\sum_{i=1}^{n}\left(f_{1}, \ldots, f_{m}\right)\left(X_{i}\right) / a_{n}\right)\right\}$ is relatively shift compact. Now the argument in the last part of the proof of Theorem 4.3(i) proves 4.10(i).

(ii) We now prove the converse. Recall that if $\rho$ in nondegenerate, $a_{n} \rightarrow \infty$ and $a_{n} / a_{n+1} \rightarrow 1$. If $\sigma(\partial A)=\sigma(\partial D)=0$, by Theorem 3.1,

$$
\begin{gathered}
\lim _{n \rightarrow \infty} P\left\{\|X\|>u a_{n+k}, X /\|X\| \in A\right\} / P\left\{\|X\|>u a_{n}, X /\|X\| \in D\right\} \\
=\sigma(A) / \sigma(D)
\end{gathered}
$$


for every $u>0$ and finite integer $k$. If $n_{t}$ is as in Corollary 3.4, we have

$$
\begin{aligned}
P\left\{\|X\|>a_{n_{1}+1},\right. & X /\|X\| \in A\} / P\left\{\|X\|>a_{n_{i}}, X /\|X\| \in D\right\} \\
& \leqslant P\{\|X\|>t, X /\|X\| \in A\} / P\{\|X\|>t, X /\|X\| \in D\} \\
& \leqslant P\left\{\|X\|>a_{n_{i}}, X /\|X\| \in A\right\} / P\left\{\|X\|>a_{n_{t}+1}, X /\|X\| \in D\right\}
\end{aligned}
$$

and taking limits as $t \rightarrow \infty$, (b) follows from (4.4).

Let now $F_{m} \subset B, m=1, \ldots$, be an increasing sequence of finite dimensional subspaces such that $\overline{\cup F_{m}}=B, F_{1}=\{0\}$, and define $C_{m}=\mu\{x$ : $\left.d\left(x, F_{m}\right)>1\right\}$. Then $C_{m} \rightarrow 0$ by tightness of $\mu \mid\{x:\|x\|>1\}$, and by (1) and (2) in the proof of 4.3(ii),

$$
\lim _{n \rightarrow \infty} n P\left\{d\left(X, F_{m}\right)>a_{n} t\right\}=C_{m} t^{-\alpha}
$$

for every $t>0$ and natural $m$. Hence, using Theorem 3.1 again,

$$
\lim _{n \rightarrow \infty} P\left\{\|X\|>a_{n+k}\right\} / P\left\{d\left(X, F_{m}\right)>u a_{n}\right\}=c_{1} u^{\alpha} / C_{m}
$$

for every $u>0$, natural $m$ and integer $k$. But (4.6) gives condition (a) exactly as condition (b) follows from (4.4).

A different proof of the direct part is possible: just apply Theorem 4.1 of de Acosta, Araujo and Giné (to appear), with the index 2 replaced by $p \in[1,2]$.

4.11. TheOREM. The following set of conditions is sufficient for $X$ to belong to the domain of attraction of a nondegenerate Gaussian p.m. $\gamma$ with covariance $\Phi$ if $B$ is of type 2 , and necessary if $B$ (separable) is the dual of a type 2 space:

$$
\lim _{t \rightarrow \infty} t^{2} P\{\|X\|>t\} / \int_{\|X\|<t}\|X\|^{2} d P=0,
$$

(b) $\quad \lim _{t \rightarrow \infty} \int_{\|X\|<t} f^{2}(X) d P / \int_{\|X\|<t} g^{2}(X) d P=\Phi(f, f) / \Phi(g, g)$

for $g \neq 0$ if $\int\|X\|^{2} d P=\infty$ and

$$
\lim _{t \rightarrow \infty} \int_{\|X\|<t} f^{2}(X-E X) d P / \int_{\|X\|<t} g^{2}(X-E X) d P=\Phi(f, f) / \Phi(g, g)
$$

for $g \neq 0$ if $\int\|X\|^{2} d P<\infty$,

(c) there exists an increasing sequence of finite dimensional subspaces $F_{m} \subset B$ such that

$$
\lambda_{m}=\limsup _{t \rightarrow \infty} \int_{\|X\|<t} d^{2}\left(X, F_{m}\right) d P / \int_{\|X\|<t}\|X\|^{2} d P>0
$$

and $\lambda_{m} \rightarrow 0$ as $m \rightarrow \infty$.

Proof. (1) Case $E\|X\|^{2}<\infty$. If $X$ is in the domain of attraction of a nondegenerate Gaussian p.m. $\gamma$ with covariance $\Phi$ and $\left\{X_{i}\right\}$ is a sequence of i.i.d. rv's with $\mathcal{L}\left(X_{i}\right)=\mathfrak{L}(X)$, then 


$$
\mathcal{L}\left(\sum_{i=1}^{n} X_{i} / n^{1 / 2}-E X\right) \rightarrow \vec{w} a \gamma
$$

for some real $a>0$ (the norming constants are necessarily of the order of $n^{1 / 2}$ by the one dimensional central limit theorem, and the natural shifts are

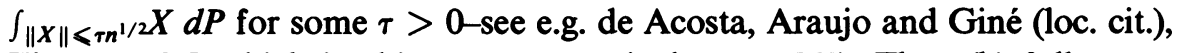
Theorem 2.5-which in this case are equivalent to $E X$ ). Then (b) follows at once from the one dimensional central limit theorem (together with a simple computation) and (a) from the hypothesis $E\|X\|^{2}<\infty$. As for (c) it is clear that $\lambda_{m} \rightarrow 0$ whenever $F_{m} \uparrow, \overline{\cup F_{m}}=B$; let us see finally that $\lambda_{m}>0$ : let $f \in B^{\prime}$ be such that $F_{m} \subset \operatorname{Ker} f$; then $d\left(x, F_{m}\right) \geqslant d(x, \operatorname{Ker} f) \geqslant|f(x)| /\|f\|$ and therefore

$$
\begin{aligned}
\int_{B} d^{2}\left(X, F_{m}\right) d P & \geqslant\|f\|^{-1} \int_{B} f^{2}(X) d P \\
& =\|f\|^{-1} \int_{B}[f(X-E X)+f(E X)]^{2} d P \\
& =\|f\|^{-1}\left[\Phi(f, f)+f^{2}(E X)\right]>0
\end{aligned}
$$

as $\Phi(f, f)>0$. Note that in this case no assumption has been made on the geometry of $B$. The proof for the converse in this case is as in Kuelbs and Mandrekar (loc. cit.), Theorem 3.1, if one invokes the central limit theorem of Hoffman-Jørgensen and Pisier (1976) instead of the classical one for Hilbert space.

(2) Case $E\|X\|^{2}=\infty$. By the Theorems 4.3 and 6.7 in de Acosta, Araujo and Gine (loc. cit.), the following conditions are sufficient in type 2 and necessary in duals of type 2 spaces for $X$ to be in the domain of attraction of a Gaussian p.m. $\gamma$ with covariance $\Phi$ and norming constants $a_{n}$ :

$$
\begin{aligned}
& \lim _{n \rightarrow \infty} n P\left\{\|X\|>t a_{n}\right\}=0 \text { for all } t>0, \\
& \sup _{n} n a_{n}^{-2} \int_{\|X\|<\varepsilon a_{n}}\left\|X-a_{n} \gamma_{n}\right\|^{2} d P<\infty,
\end{aligned}
$$

where $\gamma_{n}=\int_{\|X\| \leqslant a_{n}} a_{n}^{-1} X d P$,

$$
\lim _{m \rightarrow \infty} \sup _{n} n a_{n}^{-2} \int_{\|X\|<\varepsilon a_{n}} d^{2}\left(X-a_{n} \gamma_{n}, F_{m}\right) d P=0
$$

for some increasing sequence $\left\{F_{m}\right\}$ of finite dimensional subspaces of $B$ and some $\varepsilon>0$,

$$
\lim _{\varepsilon \downarrow 0}\left\{\begin{array}{c}
\lim \sup \\
\lim \text { inf }
\end{array}\right\}_{n \rightarrow \infty} \int_{\|X\|<\varepsilon a_{n}} f^{2}\left(X-a_{n} \gamma_{n}\right) d P=a \Phi(f, f)
$$

for every $f \in B^{\prime}$ and some $a>0$.

Assume now $X$ in the domain of attraction of a nondegenerate Gaussian 
p.m. $\gamma$ and that $B$ is the dual of a type 2 space. Then (i)-(iv) hold for some sequence $\left\{a_{n}\right\}, a_{n}>0$. On the other hand, $E f^{2}(X)=\infty$ for every nonzero $f \in B^{\prime}$, otherwise $a_{n} \sim n^{1 / 2}$ and $E\|X\|^{2}<\infty$. Hence also $E d^{2}\left(X, F_{m}\right)=\infty$. So we can use the argument in Kuelbs and Mandrekar (loc. cit.) proof of Theorem 3.1 to obtain that in (i)-(iv) the centerings $a_{n} \gamma_{n}$ can be replaced by zero. From this point on the proof that (a)-(c) hold is essentially as in that article, proof of Theorem 3.1.

Assume now that (a)-(c) hold and that $B$ is of type 2. It is enough to prove (i)-(iv) for some sequence $\left\{a_{n}\right\}$. (i) and (ii)-(iii) with $a_{n} \gamma_{n}$ replaced by zero follow as in the proof of Kuelbs and Mandrekar, hence also (i)-(iii), (ii) and (iii) with $a_{n} \gamma_{n}$ replaced by zero imply by Lemma 4.2 that $\left\{\operatorname{cPois} n \mathcal{L}\left(X / a_{n}\right)\right\}$ is relatively compact; therefore by Lemma 4.1 so is $\left\{\mathcal{L}\left(\sum_{i=1}^{n} X_{i} / a_{n}-a_{n} \gamma_{n}\right)\right\}$. But by (i) all the limit points of this sequence are Gaussian and by the general central limit theorem in de Acosta, Araujo and Giné (loc. cit., Theorem 2.10), for every convergent subsequence $\mathcal{L}\left(\sum_{i=1}^{n^{\prime}} X_{i} / a_{n},-a_{n}, \gamma_{n}\right) \rightarrow_{\mathrm{w}} \gamma^{\prime}$, and condition (iv) holds along the subsequence $\left\{n^{\prime}\right\}$ and with limit $\operatorname{cov} \gamma^{\prime}(f, f)$. Then (b) implies that $\operatorname{cov} \gamma^{\prime}=a \Phi$ for some $a \neq 0$, so that (iv) holds and $X$ is in the domain of attraction of a nondegenerate Gaussian p.m. $\gamma$ with covariance $\Phi$.

4.12. Remark. The converse part of the last theorem is also true, with obvious modifications, for cotype 2 spaces with the property that there exists a sequence of projections $\left\{\pi_{n}\right\}$ of finite dimensional range such that $\| \pi_{n}(x)-$ $x \|=d\left(x, \pi_{n}(B)\right)$ for all $x$ in $B$ (see e.g. the remark after Theorem 6.6 in de Acosta, Araujo and Giné). In particular it holds for $l_{1}$.

We end up this investigation on domains of attraction with the statement of a sufficient condition for a sample continuous process to be in the domain of normal attraction of a stable one. The proof follows easily from Theorem 4.9 in our previously mentioned article and therefore it will be omitted.

4.13. TheOREM. Let $X(s), s \in S$ compact metric, be a sample continuous process such that:

(i) each of its finite dimensional distributions belongs to the domain of normal attraction of a stable p.m. of order $\alpha<2$ in Euclidean space,

(ii) for every $\omega \in \Omega$ and $s, t \in S$,

$$
|X(s, \omega)-X(t, \omega)| \leqslant M(\omega) e(s, t)
$$

where $e(s, t)$ is a continuous distance on $S$ such that for some p.m. $\lambda$ on $S$,

$$
\lim _{\varepsilon \downarrow 0} \sup _{s} \int_{0}^{\varepsilon}[\log (1+1 / \lambda\{t \in S, e(s, t) \leqslant u\})]^{1 / 2} d u=0
$$

and $M$ is a nonnegative random variable satisfying

$$
\lim _{m \rightarrow \infty} \sup _{n} n P\left\{M>m n^{1 / \alpha}\right\}=0 .
$$


Then $X$ belongs to the domain of normal attraction of a stable p.m. of order $\alpha$ in $C(S)$.

ACKNOWLEDGements. J. Kuelbs and J. Zinn pointed out to us an error in a previous draft, and M. B. Marcus showed us examples similar to 4.8. We also acknowledge some useful conversations with A. de Acosta and with L. Le Cam.

\section{REFERENCES}

1. A. de Acosta (1970), Existence and convergence of probability measures in Banach spaces, Trans. Amer. Math. Soc. 152, 273-298.

2. __ Asymptotic behaviour of stable measures, Ann. Probability 5, 494-499.

3. A. de Acosta, A. Araujo and E. Gine (1977), On Poisson measures, Gaussian measures and the central limit theorem in Banach spaces, Advances in Probability (to appear).

4. A. de Acosta and Jorge D. Samur (1977), Infinitely divisible probability measures and the converse Kolmogorov inequality in Banach spaces, IVIC Preprint \#2, Studia Math. (to appear).

5. A. Araujo (1975), On the central limit theorem in Banach spaces (preprint).

6. (1975 a), On infinitely divisible laws in C $[0,1]$, Proc. Amer. Math. Soc. 51, 179-185; ibid 58.

7. K. L. Chung (1974), A course in probability, Academic Press, New York.

8. E. Dettweiler (1976), Grenzwertsätze für Wahrscheinlichkeitsmasse auf Badrikianschen Räumen, Z. Wahrscheinlichkeitstheorie und verw. Gebiete 34, 285-311.

9. W. Feller (1970), An introduction to probability theory and its applications, Vol. II, 2nd ed., Wiley, New York.

10. J. Hoffman-Jorgensen and G. Pisier (1976), The law of large numbers and the central limit theorem in Banach spaces, Ann. Probability 4, 587-599.

11. I. A. Ibragimov and Yu. V. Linnik (1971), Independent and stationary sequences of random variables, Noordhoff, Groningen,

12. J. Kuelbs (1973), A representation theorem for symmetric stable processes and stable measures on $H, Z$. Wahrscheinlichkeitstheorie und verw. Gebeite 26, 259-271.

13. J. Kuelbs and V. Mandrekar (1974), Domains of attraction of stable measures on a Hilbert space, Studia Math. 50, 149-162.

14. A. Kumar and V. Mandrekar (1972), Stable probability measures on Banach spaces, Studia Math. 42, 133-144.

15. L. Le Cam, Remarques sur le théorème limit central dans les espaces localement convexes, Les Probabilités sur les Structures Algébriques, Editions NRS, Paris, pp. 233-243.

16. P. Lévy (1937), Théorie de l'addition des variables aléatoires, Gauthier-Villars, Paris.

17. K. R. Parthasarathy (1967), Probability measures on metric spaces, Academic Press, New York.

18. H. Schaefer (1973), Topological vector spaces, Springer-Verlag, Berlin and New York.

Department of Economics, University of Chicago, Chicago, Illinois 60637

Departamento de Matemáticas, I.V.I.C., Apartado 1827, Caracas 101, Venezuela 